\title{
Levels of Chromospheric and Coronal Activity in Sun-like Stars and Various Types of Dynamo Waves
}

\author{
E.A. Bruevich ${ }^{1}$, M.M. Katsova ${ }^{1}$, D.D. Sokolov ${ }^{2}$ \\ ${ }^{1}$ Lomonosov Moscow State University, Sternberg Astronomical Institute, \\ Universitetsky pr., 13, Moscow 119992, Russia \\ ${ }^{2}$ Lomonosov Moscow State University, Faculty of Physics, \\ Vorob'evy gory, Moscow 119992, Russia \\ e-mail: red-field@yandex.ru
}

\begin{abstract}
We analyze the X-ray emission and chromospheric activity of Sun-like stars of F, G, and K spectral classes (late-type stars) studied in the framework of the HK project. More powerful coronas are possessed by stars displaying irregular variations of their chromospheric emission, while stars with cyclic activity are characterized by comparatively modest X-ray luminosities and ratios of the X-ray to bolometric luminosity $L_{X} / L_{b o l}$. This indicates that the nature of processes associated with magnetic-field amplification in the convective envelope changes appreciably in the transition from small to large dynamo numbers, directly affecting the character of the $(\alpha-$ $\Omega$ ) dynamo. Due to the strong dependence of both the dynamo number and the Rossby number on the speed of axial rotation, earlier correlations found between various activity parameters and the Rossby number are consistent with our conclusions. Our analysis makes it possible to draw the first firm conclusions about the place of solar activity among analogous processes developing in active late-type stars.
\end{abstract}

Key words. Sun-like stars: HK project: chromospheric activity: X-ray emission.

\section{Introduction}

The first systematic investigations of solar-type activity in other stars were begun in the middle of the 1960s by Wilson at Mt. Wilson Observatory, and have continued since that time in the framework of the so called "HK 
project" [1, 2]. Observational studies of chromospheric activity include determinations of the ratio of the central fluxes of the $\mathrm{Ca}$ II $\mathrm{H}$ and $\mathrm{K}$ lines (3968 Aand $3934 \AA$, respectively) to the flux in the nearby continuum (4001 Aand $3901 \AA$ ) - the quantity $S_{C a I I}$ (the mean ratio for the two lines). This approach has provided a uniform description of levels of chromospheric activity and made it possible to monitor such activity over several decades. Several dozen fairly bright stars with spectral types from F2 to M2, in which it was believed solar-type magnetic activity could develop, were chosen for study. Beginning in 1977, the number of program stars grew to 111, and this database became fundamental for investigations of chromospheric variability on time scales from decades down to the rotational periods of the stars. The quantity $S_{\text {CaII }}$ (further $\mathrm{S}$ ) proved to be a good indicator of chromospheric activity [3]. Objects with both large and small values of $S$ are encountered among stars with similar spectral types. Baliunas et al. [3] attributed these differences in the level of chromospheric activity primarily to differences in age, at least in the case of single stars. The mean (for the given interval of effective temperatures) value of $\mathrm{S}$ varies somewhat with spectral type. It has now become clear that the processes giving rise to the complex of activity phenomena manifest in the interiors-convective zones-of stars encompass virtually the entire atmosphere from the photosphere to the corona. The first results on variations of the optical continuum of stars in the HK project over roughly the last ten years are presented in [4]; results for some BY Dra stars (spotted red dwarfs) are presented in $[5,6]$. In general, these data testify that the photospheric activity of these stars resembles that of the Sun, but, in some cases, the relative areas occupied by star spots are two to three orders of magnitude larger than on the Sun at the solar-cycle maximum. Data that can be used to compare the properties of activity in different levels of the outer atmospheres of specific stars and the Sun are already available.

Long-term, uniform data sets on the coronal fluxes of specific late-type stars are not yet available, except for the Sun. However, measurements of the soft X-ray emission of more than 1000 active late-type stars have been made. These are primarily data obtained for nearby, bright stars by the ROSAT satellite at $0.1-2.4 \mathrm{keV}[7,8]$. The ROSAT data confirmed and expanded the general results obtained by the Einstein observatory concerning the dependence of the X-ray luminosity on the speed of axial rotation, at least for main-sequence stars; the saturation of the X-ray luminosity relative to the bolometric luminosity $L_{X} / L_{b o l}$ for red dwarfs (discovered by Vilhu and Walter [9]); and the presence of high-temperature patches in the coronas 


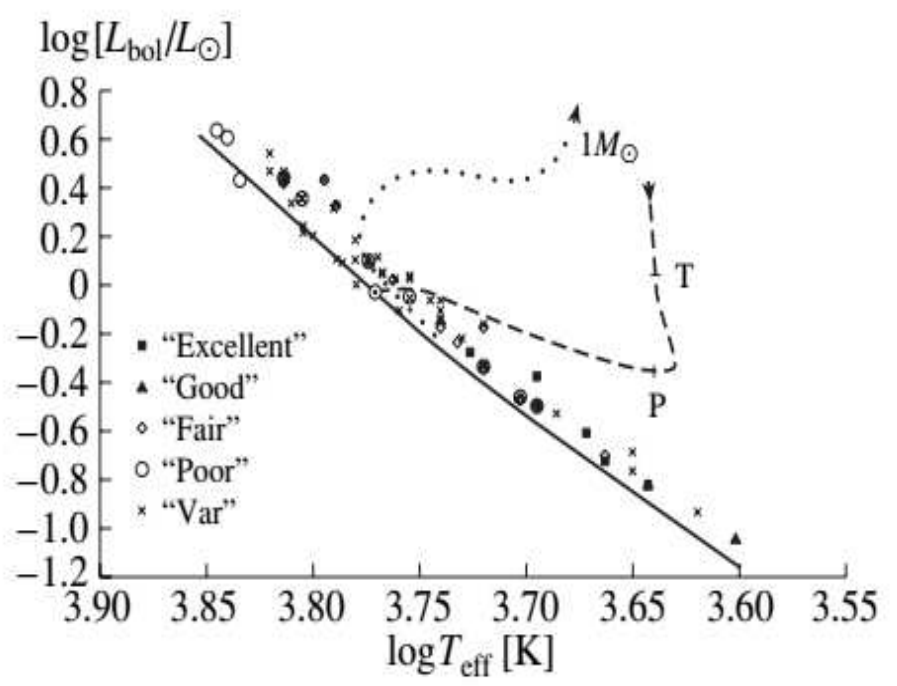

Figure 1: Position in the Hertzsprung - Russel diagram of late-type stars with chromospheric activity included in the HK project. The various symbols correspond to different manifestations of chromospheric activity, from chaotic irregular variations (crosses) to cyclic activity (filled squares). The solid curve shows the main sequence; the dashed curve shows the track for the arrival to the main sequence of a solar-mass star from the evolutionary status of $\mathrm{T}$ Tauri $(\mathrm{T})$ through the post- $\mathrm{T}$ Tauri stage $(\mathrm{P})$. The dotted curve shows the departure of a star of the same mass from the main sequence. The position of the Sun is marked with a solar sign. A number of stars with the same spectral type are superposed, so that the number of stars in the diagram is fewer than the number in Tables $1-4$.

of some late-type stars [10]. Important information has been obtained for active latetype stars in RS CVn binary systems, enabling in a number of cases a better understanding of the regularities observed for active processes on single late-type stars. An analysis of the X-ray emission of stellar coronas based on Einstein data was presented by Katsova et al. [11], who determined the densities at the bases of the uniform coronas of dwarfs of various spectral types. The coronas with the highest densities are possessed by the most active red dwarfs, with spectral types K5-M3; their coronal densities are an order of magnitude higher than the corresponding solar value. The X-ray emission 

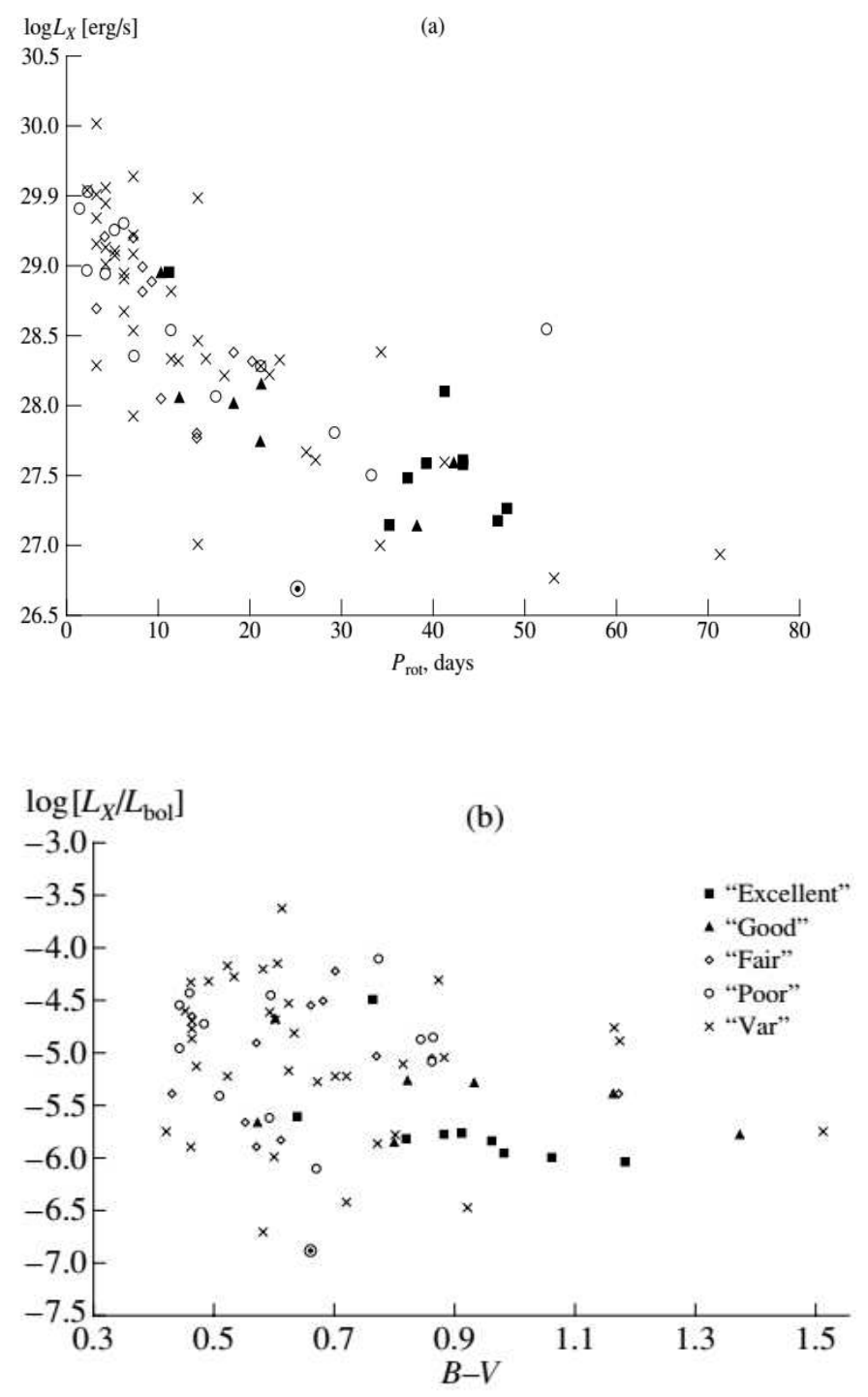

Figure 2: (a) X-ray luminosity as a function of the period of axial rotation for HK project stars (from ROSAT data). (b) Ratio of the X-ray to the bolometric luminosity for HK project stars as a function of B-V. Notation is the same as in Fig. 1. 
of HK project stars detected by ROSAT was considered by Hempelmann et al. [12], who compared the X-ray emission of late-type stars with constant levels of Ca II line emission and with regular and irregular (chaotic) longterm variations of this emission. They found that the distribution of X-ray fluxes associated with the stellar coronas is appreciably different for stars with variable Ca II line fluxes: on average, stars with irregular chromospheric activity have higher X-ray fluxes. Hempelmann et al. [12] attempted to relate this result to a dependence of coronal activity on the Rossby number, and to the possible existence of a Maunder minimum at the current epoch for some quiescent late-type stars. Uniform X-ray catalogs of the nearest bright stars are now available $[7,8]$, as well as the results of new analyses of long-term chromospheric and photospheric activity.

Therefore, it is valuable to investigate the coronal activity of HK project stars with different types of cycles and compare the results with the main expectations of dynamo theory. This can provide information about the effectiveness of the dynamo mechanism on F-K stars with differing levels of activity.

\section{Chromospheric activity and position of a star on the Hertzsprung - Russell diagram}

Chromospheric activity and position of a star on the Hertzsprung - Russell diagram In most stars with variable Ca II line emission, the calcium-line flux shows modulations with the period of rotation of the star. This rotational modulation virtually disappears for stars of similar spectral types with the smallest and largest values of S, due to either a lack of active regions on these stars or, on the contrary, the presence of a very large number of active regions more or less uniformly distributed over the surface (if the stellar rotational axis is perpendicular to the line of sight, this implies a uniform distribution in longitude). Observations carried out over the past 35 years have enabled the detection of long-term variations of this activity index for selected variable stars. Roughly one third of the studied stars show no periodicities in these variations (this type of chromospheric variability is designated after [3] as "Var"). Long-term periodicity in variations of $\mathrm{S}$ were directly detected in 50 of the 111 HK-project stars. Applying spectral analyses to the long-term data on chromospheric activity made it possible to determine for each star 


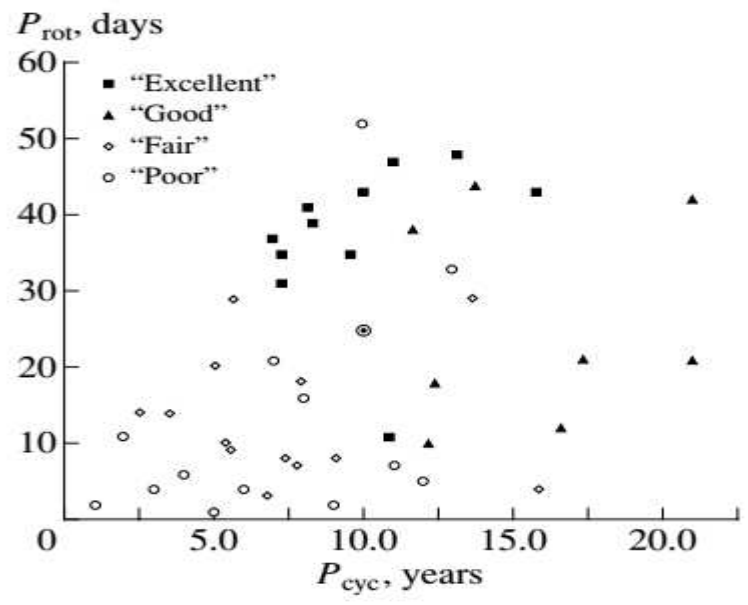

Figure 3: Periods of axial rotation and cycle durations for HK project stars with more or less well defined activity cycles. Notation is the same as in Fig.1.

the degree to which cycles analogous to the solar cycle were present and well defined. The periods for the cycles were from several years to decades. Using formal statistical criteria, Baliunas et al. [3] categorized stars into four groups according to the degree to which their chromospheric-activity cycles were well defined: "Excellent", "Good", "Fair" and "Poor". This division was carried out based on the probability that the periodicity for stars in a given group was not associated with Gaussian noise. A quantitative index for this division is the false alarm probability (FAP), or the probability that the observed behavior is the result of Gaussian noise; for "Excellent" cycles FAP $<10^{-9}$, for "Good" cycles $10^{-9}<$ FAP $<10^{-5}$, for "Fair" cycles $10^{-5}<$ FAP $<10^{-2}$, and for "Poor" cycles $10^{-2}<\mathrm{FAP}<10^{-1}$. A standard statistical treatment indicates that the critical value of the FAP for which the null hypothesis of the presence of cyclicity can be rejected is about 0.05. Formally, this means that one out of 20 cases that are in fact due to Gaussian noise will be taken to represent cyclic behavior. However, between these extreme cases of pure Gaussian noise and strictly periodic variations, there are many different possible types of variability that formally contain some periodic components, 


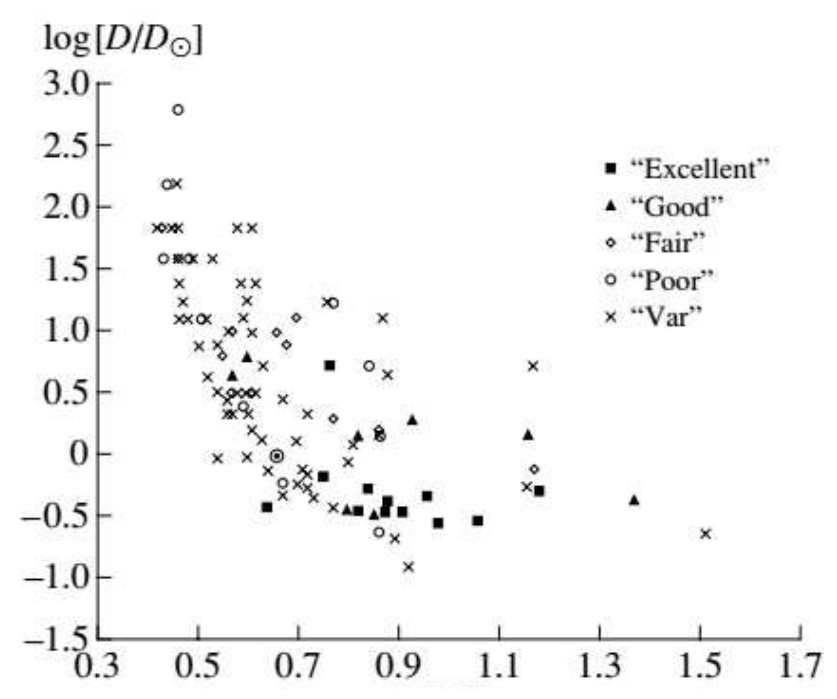

Figure 4: Variation of the dynamo number (relative to the solar value) as a function of B-V for HK project stars. Notation is the same as in Fig. 1.

which can be revealed by Fourier analysis. In some cases, it is also important to accurately exclude harmonics. Therefore, a trustworthy identification of cycles requires the application of not one but several methods and criteria. Recent progress in finding solutions to this problem has come about with the development of wavelet analysis, which enables the analysis of non-uniform time series. In summary, the FAP can serve as a certain relative measure of the extent to which cyclic variations are well defined. The broad range for the "Good" group $\left(10^{-9}<\mathrm{FAP}<10^{-5}\right)$ essentially means that there is already a watershed here dividing cycles that can be distinguished with certainty from cases with more complex activity on time scales of several years. In their analysis, Baliunas et al. [3] note that cycles are more easily distinguished for $\mathrm{K}$ stars and for $\mathrm{G}$ stars with small $\mathrm{S}$ values, while the chromospheric activity of some F stars is higher, but more chaotic (cyclicity is virtually absent). This result can be represented pictorially by plotting the HK project objects with chromospheric activity on a Hertzsprung - Russell diagram. We determined the starss $\mathrm{T}^{\mathrm{TM}}$ bolometric luminosities using the fundamental parameters given in $[13,14]$ (see Tables $1-4)$. Figure 1 illustrates the behavior of the stars with chromospheric activity by plotting their bolometric luminosities 
(in solar units) $\log L_{b o l} / L_{\odot}$ ( as a function of their effective temperatures $T_{\text {eff }}$. Here, we have drawn the conventional zero-age main sequence and two evolutionary tracks demonstrating the arrival to and departure from the main sequence of solar-mass stars [15].

We can see that these stars are located fairly close to the main sequence. This reflects the fact that the youngest stars, with spectral types later than F0, and evolved objects were not considered either in the HK project or in the current paper. However, there is some scatter in the ages of the stars studied, which may affect their activity levels to some extent. Our independent analysis and the analysis of [3] show that the cyclicity of longterm chromospheric variations begins to be manifest in stars of spectral type G0, and is clearly defined in spotted $\mathrm{K}$ stars, while this activity is irregular in F stars. Recent data on chromospheric activity and new observations of longterm variations in the optical continuum of the HK project stars enable us to refine this conclusion. Beginning with [16], the idea has been developed that all these late-type stars can be divided into two groups according to their activity levels. This division becomes more obvious when considering the calcium emission fluxes. In other words, in place of the index S, the flux of a star in both calcium emission lines normalized to the bolometric luminosity, $\log R_{H K}^{\prime}$ [17], was introduced as a measure of activity, and began to be used in subsequent studies. In relations plotting $\log R_{H K}^{\prime}$ as functions of spectral type or other stellar parameters, two groups can clearly be distinguished, with stars with well defined cycles being less active. This tendency has been traced in several studies, in particular in [4], which presents the results of ten years of optical-continuum observations of a number of HK project stars. It has also become possible to compare long-term activity at the chromospheric and photospheric levels, which increases our ability to determine the extent to which variations display well defined cyclicity.

Note that the difference in the activity levels of these two groups of stars depends on their speeds of axial rotation, which also indirectly reflects the influence on rotation of several factors, first and foremost age. All these considerations lead us to believe that cycles can be distinguished with a high degree of certainty only for stars in the "Excellent" group. We have already noted that stars in the "Good" group have FAPs that cover a very wide range (four orders of magnitude). This means that this group includes both stars with well defined cycles (with periods, however, that have not been strictly maintained over the forty years of observations) and stars with irregular variations of their chromospheric and photospheric emission. Note 
that Baliunas et al. [3] ascribe only 13 stars, including the Sun, to the "Excellent" group; well defined cycles are observed in only a few of the 8 stars in the "Good" group.

\section{X-ray emission and cyclic activity of late- type stars}

To analyze the coronal emission of late-type stars, we used ROSAT observations of more than 1500 nearby, bright main-sequence stars and subgiants, contained in the two X-ray catalogs of Hunsch et al. [7, 8]. Both catalogs present measurements in the 0.1-2.4 keV range carried out either as part of the ROSAT all-sky survey or during directed observations. We were able to find X-ray flux measurements for 78 of the HK project stars. We used the data for these objects from [7, 8] reduced to a unified system. In several cases when the total X-ray flux from a binary system was registered (HD 131156AB, HD 165341AB, HD 201091, and HD 201092, HD 219834AB), we ascribed this flux equally to both components if their speeds of axial rotation were essentially the same. In two such cases, we divided the total flux of the binary in proportion to the rotational speeds of the two stars. Note also that the X-ray luminosity of HD 81809 may be overestimated, since this is a spectroscopic binary. The X-ray luminosities $L_{X}$ for the HK project stars (when observed) are presented in Tables 1,2 (for stars with more or less regular chromospheric activity) and Tables 3,4 (for stars with chaotic variability). These tables also list a number of other physical characteristics of these stars: their color indices, spectra, periods of axial rotation $P_{\text {rot }}[18]$, bolometric luminosities $L_{b o l}$, etc. For the Sun, we present the X-ray luminosity in the ROSAT band for epochs of high activity $\left(L_{X}=5 \cdot 10^{26} \mathrm{erg} / \mathrm{s}\right)$ [19]. Figure 2 a presents the X-ray luminosities of the HK project stars and the Sun together with characteristics of their chromospheric activity as functions of their rotational periods. The main tendency for more rapidly rotating latetype stars to possess higher X-ray fluxes- first discovered in Einstein data [20]-can clearly be traced here. Recall that the stars in Fig. 2 are near the main sequence (Fig. 1), for which the relationship between X-ray luminos-

ity and rotational speed is undoubted. The presence of some scatter among the points, especially the deviation from the lower envelope toward larger luminosities, is due to the fact that some stars, even those near the Sun, are 
younger than the Sun. The influence of age is manifest first and foremost in the rotation speed: younger stars have shorter rotational periods. However, the shift of the points in Fig. 2a due to the influence of age is not strictly along the $\mathrm{X}$ axis; this becomes clear, for example, if we join the points from [10] for $G$ stars with different ages. This means that, even if we consider a single rotational period, younger objects are higher in this diagram than stars that are older or the same age as the Sun. The uniform data considered here clearly show a result noted earlier in [12]: the X-ray luminosities of stars with irregular, chaotic activity are higher than those of objects with well established activity cycles. One exception is the rapidly rotating star V2292 Oph (HD 152391; $P_{\text {rot }}=11$ days), whose unusual chromospheric and photospheric variations were recently noted in [21].

Analysis of the X-ray data for the groups of stars with different degrees of cyclicity shows that stars within a single group tend to cluster in a log $L_{X}-P_{\text {rot }}$ diagram. This is especially true of the eight stars in the Excellent group and two stars in the Good group with clearly defined cycles. These stars, with spectral types G7-K7 (except for HD 81809, whose spectral type is close to that of the Sun), are clustered near $\log L_{x} \approx 27.5$ and $P_{\text {rot }} \approx 40$ days. They also occupy a very small region in the $\log L_{b o l}-P_{\text {rot }}$ diagram with $\log L_{X} / L_{b o l} \approx-5.7$.

Stars with other activity parameters, which rotate more rapidly, and which have poorly defined cycles demonstrate less strong clustering; their $\mathrm{X}$-ray luminosities are factors of three to one hundred higher than those of stars with better defined cyclicity. Similar behavior can be seen in Fig. 2b, which plots the ratio of the X-ray to the bolometric luminosity as a function of the $\mathrm{B}-\mathrm{V}$ color index. We can see that stars with well defined cycles have roughly the same ratio $\log L_{X} / L_{b o l} \approx-5.7$. At the same time, for most stars with irregular chromospheric activity, this ratio covers the wide range log $L_{X} / L_{b o l}=-4.2$ to -6.0 . These values are far from the limiting value for X-ray saturation for active red dwarfs and components of RS CVn binary systems, $\log L_{X} / L_{b o l} \approx-3.0$. The mean value of $\log L_{X} / L_{b o l}$ for the Sun is roughly a factor of 30 lower than for most of the HK project stars considered here. This is primarily due to the different ages for these stars and the Sun. In addition, this could reflect individual properties of the Sun's activity compared to the analogous processes developing on other stars. Note that the Sun is the most rapid rotator among the stars with well defined cycles (with the exception of V2292 Oph, discussed above). It is interesting to ask what determines the duration of the cycle of an individual star. The available observations 
made over 35 years contain only limited information about this question. Figure 3 compares the cycle durations and rotational periods for the groups of stars with differing degrees of cyclicity. In a number of cases, a statistical analysis leads to poorly defined, long-term variations being represented as a superposition (as a rule, of two) periodic components; clearly, such cycle durations should be treated with caution. Therefore, we have presented two pairs of values in Fig. 3 (the duration of the cycle and its harmonic) only for the three stars HD 78366, HD 114710 ( $\beta$ Com), and HD 149661 (V2133 Oph), whose main cycles are characterized as "Good". Stars with well defined cycles rotate slowly, and the durations of their cycles are from 7 to 17 years. In con trast to the earlier opinion that well defined cycles usually have durations of 10-11 years, Fig. 3 shows that the scatter in the durations is appreciable. As noted above, V2292 Oph has an exception cycle, as can also be seen in this figure. The durations of the cycles of rapidly rotating stars with poorly defined periods for their long-term variations cover a wide range from 2.8 to roughly 20 years, but nearly half of them have "cycles" shorter than 8 years. This provides evidence that decreasing the rotational speed not only leads to the formation of a stable cycle, but that the duration of this cycle will exceed the values typical of rapidly rotating stars. The information discussed above can be used to address the question of the place of solar activity among similar processes occurring on late-type stars. Here, we should bear in mind several important factors. The Sun has the earliest spectral type in the group of stars with well defined cycles. The chromospheric activity of the Sun as a star is similar to the mean activity level for stars of this group, as is indicated by a comparison of the flux in the Ca II $\mathrm{H}$ and $\mathrm{K}$ lines ; this can be seen in Fig. 8a of [4]. On the other hand, the X-ray luminosity of the Sun is appreciably smaller than those of other stars with cycles, as is clearly reflected by the ratio of its X-ray to its bolometric luminosity. In addition, the Sun rotates appreciably more rapidly than the remaining stars with well defined cycles. It is possible that the Sun is located near the boundary where chaotic, irregular activity is transformed into more regular, cyclic behavior. This could be an important argument in searching for the origin of the Maunder minimum. Thus, we can see that stars with well defined activity cycles and those whose activity is fairly irregular form two clusters in our diagrams. Well defined cycles correspond to longer durations of the activity cycles, longer rotational periods, and lower X-ray fluxes; i.e., lower magnetic-field strengths. This last correlation can also be seen in the figure presented in [23], although it is not specially discussed in that work. 
This last conclusion may, at first glance, seem paradoxical from the point of view of dynamo theory, which explains both the magnetic-field strength and the cycle duration in the framework of a single mechanism for the generation of the magnetic field. Below, we will demonstrate that this correlation can fit into the stellar dynamo mechanism.

\section{Parameters of stellar cycles and the Parker dynamo}

The explanation of the origin of stellar cycles in the framework of dynamo theory proposed by Parker in 1955 can be reduced to the following. The poloidal component of the large-scale magnetic field of a star, which can be expressed in terms of the azimuthal component of the vector potential of the magnetic field $\mathrm{A}$, is wound up by differential rotation, leading to the formation of an azimuthal component of the magnetic field B. In turn, the helicity of the convective flows acts on the azimuthal field component, giving rise to a poloidal magnetic field and closing the cycle of self-excitation. This process can be described quantitatively using the Parker dynamo equations, which, in the simplest case, have the form

$$
\begin{gathered}
\frac{\partial A}{\partial t}=\alpha B+\frac{\partial^{2} A}{\partial \theta^{2}}, \\
\frac{\partial B}{\partial t}=-D G \cos \theta \frac{\partial A}{\partial \theta}+\frac{\partial^{2} B}{\partial \theta^{2}},
\end{gathered}
$$

where $\theta$ is latitude, and

$$
G=r^{-1} \frac{\partial \Omega}{\partial r}
$$

is the radial gradient of the angular velocity in units of its maximum value. The quantity $\alpha$ is measured in units of its maximum value. The dimensionless number $\mathrm{D}$, called the dynamo number, characterizes the intensity of sources of magnetic field generation. This number is expressed in terms of parameters characterizing the hydrodynamics of the convective envelope; in the simplest case, we can use the approximate expression 
Table 1: Stars with regular chromospheric actifity

\begin{tabular}{|c|c|c|c|c|c|c|c|}
\hline $\mathrm{HD}$ & B-V & Spectrum & $\log L_{X}, \mathrm{erg} / \mathrm{s}$ & $P_{\text {rot }}, \mathrm{d}$ & $P_{c y c}, \mathrm{y}$ & $\log L_{b o l}, \operatorname{erg} / \mathrm{s}$ & $D / D_{\odot}$ \\
\hline & & & "Excellent" & & & & \\
\hline Sun & 0.66 & $\mathrm{G} 2 \mathrm{~V}$ & 26.70 & 25 & 10.0 & 33.58 & 1.00 \\
\hline 4628 & 0.88 & $\mathrm{~K} 4(\mathrm{~K} 2 \mathrm{~V})$ & 27.59 & 39 & 8.4 & 33.33 & 0.41 \\
\hline 10476 & 0.84 & Kl V & - & 35 & 9.6 & 33.37 & 0.51 \\
\hline 16160 & 0.98 & $\mathrm{~K} 3 \mathrm{~V}$ & 27.26 & 48 & 13.2 & 33.22 & 0.27 \\
\hline 26965 & 0.82 & $\mathrm{Kl} \mathrm{V}$ & 27.61 & 43 & 10.1 & 33.38 & 0.34 \\
\hline 32147 & 1.06 & $\mathrm{~K} 5 \mathrm{~V}$ & 27.18 & 47 & 11.1 & 33.18 & 0.28 \\
\hline 81809 & 0.64 & $\mathrm{G} 2 \mathrm{~V}$ & 28.10 & 41 & 8.2 & 33.59 & 0.37 \\
\hline 103095 & 0.75 & G8 VI & 31 & 7.3 & 33.44 & 0.65 & \\
\hline 152391 & 0.76 & G7 V & 28.94 & 11 & 10.9 & 33.42 & 5.17 \\
\hline 160346 & 0.96 & $\mathrm{~K} 3 \mathrm{~V}$ & 27.48 & 37 & 7.0 & 33.25 & 0.46 \\
\hline 166620 & 0.87 & $\mathrm{~K} 2 \mathrm{~V}$ & 43 & 15.8 & 33.34 & 0.34 & \\
\hline 201091 & 1.18 & $\mathrm{~K} 5 \mathrm{~V}$ & 27.15 & 35 & 7.3 & 33.08 & 0.51 \\
\hline 219834B & 0.91 & $\mathrm{~K} 2 \mathrm{~V}$ & $\begin{array}{l}27.58 \\
\text { "Good" }\end{array}$ & 43 & 10.0 & 33.31 & 0.34 \\
\hline 3651 & 0.85 & $\mathrm{~K} 0 \mathrm{~V}$ & & 44 & 13.8 & 33.36 & 0.32 \\
\hline 78366 & 0.60 & G0 V & 28.95 & 10 & 12.2 & 33.60 & 6.25 \\
\hline 114710 & 0.57 & G0 V & 28.06 & 12 & 16.6 & 33.72 & 4.34 \\
\hline 115404 & 0.93 & $\mathrm{~K} 1 \mathrm{~V}$ & 28.02 & 18 & 12.4 & 33.28 & 1.93 \\
\hline 149661 & 0.82 & $\mathrm{~K} 0 \mathrm{~V}$ & 28.16 & 21 & 17.4 & 33.38 & 1.42 \\
\hline 156026 & 1.16 & $\mathrm{~K} 5 \mathrm{~V}$ & 27.75 & 21 & 21.0 & 33.13 & 1.42 \\
\hline 201092 & 1.37 & $\mathrm{~K} 7 \mathrm{~V}$ & 27.15 & 38 & 11.7 & 32.93 & 0.43 \\
\hline $219834 \mathrm{~A}$ & 0.80 & G5 IV-V & $\begin{array}{l}27.59 \\
\text { "Fair" }\end{array}$ & 42 & 21.0 & 33.40 & 0.35 \\
\hline 1835 & 0.66 & $\mathrm{G} 2 \mathrm{~V}$ & 28.99 & 8 & 9.1 & 33.54 & 9.77 \\
\hline 18256 & 0.43 & F5 V & 28.69 & 3 & 6.8 & 34.05 & 69.44 \\
\hline 20630 & 0.68 & G5 V & 28.89 & 9 & 5.6 & 33.50 & 7.72 \\
\hline 26913 & 0.70 & G8 V & 29.20 & 7 & 7.8 & 33.49 & 12.76 \\
\hline 82885 & 0.77 & G8 IV-V & 28.38 & 18 & 7.9 & 33.42 & 1.93 \\
\hline 100180 & 0.57 & F7 V & 27.77 & 14 & 3.6 & 33.72 & 3.19 \\
\hline 154417 & 0.57 & $\mathrm{~F} 8 \mathrm{~V}$ & 28.82 & 8 & 7.4 & 33.72 & 9.77 \\
\hline 157856 & 0.46 & F5 V & 29.21 & 4 & 15.9 & 33.93 & 39.06 \\
\hline 161239 & 0.65 & G6 V & 29 & 5.7 & 33.58 & 0.74 & \\
\hline $165341 \mathrm{~A}$ & 0.86 & $\mathrm{~K} 0 \mathrm{~V}$ & 28.31 & 20 & 5.1 & 33.35 & 1.56 \\
\hline 187691 & 0.55 & $\mathrm{~F} 8 \mathrm{~V}$ & 28.05 & 10 & 5.4 & 33.75 & 6.25 \\
\hline 190007 & 1.17 & $\mathrm{~K} 4 \mathrm{~V}$ & 27.8113 & 29 & 13.7 & 33.10 & 0.74 \\
\hline 190406 & 0.61 & G1 V & 27.80 & 14 & 2.6 & 33.64 & 3.19 \\
\hline
\end{tabular}


Table 2: Table 1.(Contd.) Stars with regular chromospheric actifity

\begin{tabular}{ccclclcl}
\hline HD & B-V & Spectrum & $\log L_{X}, \mathrm{erg} / \mathrm{s}$ & $P_{r o t}, \mathrm{~d}$ & $P_{c y c}, \mathrm{y}$ & $\log L_{b o l}, \mathrm{erg} / \mathrm{s}$ & $D / D_{\odot}$ \\
\hline & & & "Poor" & & & & \\
3229 & 0.44 & F2 V (F IV) & 29.53 & 2 & 4.9 & 33.0 & 156.25 \\
37394 & 0.84 & K1 V & 28.55 & 11 & 3.6 & 33.37 & 5.17 \\
76572 & 0.43 & F3 V & & 4 & 7.1 & 34.05 & 39.06 \\
82443 & 0.77 & K0 V & 29.3 & 6 & 2.8 & 33.42 & 17.36 \\
111456 & 0.46 & F6 V & 29.41 & 1 & 7.0 & 33.94 & 625.0 \\
120136 & 0.48 & F7 V & 28.95 & 4 & 11.6 & 33.87 & 39.06 \\
155885 & 0.86 & K1 V & 28.28 & 21 & 5.7 & 33.35 & 1.42 \\
176051 & 0.59 & G0 V & 28.06 & 16 & 100 & 33.69 & 2.44 \\
182101 & 0.44 & F6 V & 28.97 & 2 & 5.1 & 34.0 & 156.25 \\
188512 & 0.86 & G8 IV & 28.56 & 52 & 4.1 & 33.35 & 0.23 \\
194012 & 0.51 & F5 V & 28.36 & 7 & 16.7 & 33.82 & 12.76 \\
206860 & 0.59 & G0 V & 29.25 & 5 & 6.2 & 33.69 & 25.00 \\
224930 & 0.67 & G3 V & 27,51 & 33 & 10.2 & 33.52 & 0.57 \\
\hline
\end{tabular}




$$
\frac{D}{D_{\odot}}=\left(\frac{R}{R_{\odot}}\right)^{3}\left(\frac{h_{\odot}}{h}\right)\left(\frac{V_{\text {conv }}}{V_{\text {conv }}}\right)^{2}\left(\frac{\Omega}{\Omega_{\odot}}\right)^{2},
$$

where $\mathrm{R}$ is the radius of the zone of magnetic-field generation, $\mathrm{h}$ is the scale height, $\Omega$ is the angular velocity of the stellar axial rotation, and $V_{\text {conv }}$ is the convective velocity. Here, when calculating $\mathrm{G}$, we exchanged $\frac{\partial \Omega}{\partial r}$ with $\frac{\Omega}{r}$ and estimated $\alpha$ using the so-called Krause formula (see, for example, [24]).

When deriving the Parker dynamo equations, it is assumed that the convective zone, indeed, forms a sort of envelope. If the convective zone is very narrow, then the term corresponding to radial diffusion of the magnetic field must be kept in equations (1) and (2). In the case of very narrow, vanishingly thin convective zones, this term becomes dominant and disrupts the dynamo action, so that the magnetic field decays. If the convective zone occupies the entire star, the generation of magnetic field is possible, but it has an appreciably different character than in the case of a convective envelope. In particular, the generation sources must usually have appreciably higher intensities than in the case of a convective zone with moderate thickness. The question of the character and origin of the change in the type of dynamo in the presence of a degenerate point radiative core remains poorly studied theoretically. We emphasize that our stars have convective zones of moderate thickness.

Applying (3) to specific late-type stars, we should note that the first three multiplicative factors on the right-hand side compensate each other to a large extent. For example, if we use classical models for the convective zones, the products of these three factors for stars from F5 to M3 differ by only a factor of two. Thus, if we do not consider the limiting cases of stars with very thin convective zones and the latest $M$ stars with complete convection, the dynamo number can be taken to be proportional to the square of the angular velocity of rotation of the star. Traditionally, the behavior of periods of stellar activity has been analyzed using the Rossby number. Since both the dynamo numbers and the Rossby numbers of our stars are primarily determined by the angular velocity of rotation (relative to that of the Sun), the two parametrizations are similar in practice. However, conceptually, the dynamo number characterizes the sources of field generation in the dynamo mechanism and appears in the Parker equations explicitly, so that we will use this quantity in our analysis. We estimated the dynamo numbers using (3), and present the dependences of parameters of the stellar cycles on the 
dynamo number (in units of the solar value) in Fig. 4. We can see that well defined cycles correspond to comparatively low dynamo numbers D and modest magnetic-field strengths. When the dynamo number increases, the magnetic-field strength also increases, as seems natural (increased intensity of the generator leads to a growth in the generated field). However, the degree of organization of the cycle decreases, so that stars with very well defined cycles give way to stars with higher, less constant levels of activity and weakly defined cycles.

To explain this correlation, we turn to the properties of Eqs. (1) and (2). They have solutions in the form of travelling waves, called dynamo waves, whose propagation through the convective zone reflects the phenomenon of stellar activity. The dispersion relation linking the complex Eigen number of these equations-i.e., the growth rate and cycle frequency-and the dynamo wavelength separates out the dynamo wave with the maximum growth rate. It is natural to suppose that it is this wave that determines the formation of waves of stellar activity, and that precisely its parameters are inherited at the stage of evolution of the magnetic field when non-linear effects cease the exponential growth of the dynamo-wave amplitude. It is not difficult to convince oneself that the wavelength and period of this distinguished dynamo wave decrease with growth in D. Precisely this tendency can be seen in Fig. 4 (see [23]).

The decrease in the wavelength of the dynamo wave with growth in the dynamo number for a fixed convective-zone radius means that more and more half-waves of magnetic activity begin to be present between the poles and equator. It is natural to expect that, in the nonlinear regime, each of these half-waves lives a comparatively independent life; in the language of the theory of dynamical systems, Eqs. (1) and (2) describing the magnetic field as a distributed system with an infinite number of degrees of freedom can be approximately reduced to a certain dynamical system. The number of variables (degrees of freedom) in this system is determined by the number of half-waves present in the direction of propagation of the dynamo wave.

This effective number of degrees of freedom increases with the dynamo number. Similar phenomena are typical of a wide circle of problems in nonlinear dynamics. This was first noted by Hopf in his formulation of a scenario for the transition from laminar to turbulent flow. The growth in the number of degrees of freedom occurs in a discrete fashion, making it tempting to interpret the existence of discrete groups with different characters of stellar activity-though, of course, distinguished somewhat arbitrarily-as a reflection 
Table 3: Stars with irregular (chaotic) chromospheric actifity

\begin{tabular}{|c|c|c|c|c|c|c|}
\hline HD & $\mathrm{B}-\mathrm{V}$ & Spectrum & $\log L_{X}, \mathrm{erg} / \mathrm{s}$ & $P_{r o t}, \mathrm{~d}$ & $\log L_{b o l}, \mathrm{erg} / \mathrm{s}$ & $D / D_{\odot}$ \\
\hline 3443 & 0.72 & G5 V & & 30 & 33.77 & 0.69 \\
\hline 3795 & 0.70 & G3 V & & 33 & 33.49 & 0.57 \\
\hline 6920 & 0.60 & F8 V & 29.47 & 14 & 33.66 & 3.19 \\
\hline 9562 & 0.64 & $\mathrm{G} 2 \mathrm{~V}$ & & 29 & 33.59 & 0.74 \\
\hline 10700 & 0.72 & G8 V & 27.00 & 34 & 33.47 & 0.54 \\
\hline 10780 & 0.81 & K0 V & 28.34 & 23 & 33.40 & 1.18 \\
\hline 12225 & 0.62 & G1 V & 28.46 & 14 & 33.63 & 3.19 \\
\hline 13421 & 0.56 & F8 V & & 17 & 33.73 & 2.16 \\
\hline 16673 & 0.52 & $\mathrm{~F} 8 \mathrm{~V}$ & 28.54 & 7 & 33.79 & 12.76 \\
\hline 17925 & 0.87 & K0 V & 28.08 & 7 & 33.34 & 12.76 \\
\hline 22049 & 0.88 & $\mathrm{~K} 2 \mathrm{~V}$ & 28.32 & 12 & 33.33 & 4.34 \\
\hline 22072 & 0.89 & G7 V & & 55 & 33.32 & 0.21 \\
\hline 23249 & 0.92 & K0 V & 26.95 & 71 & 33.30 & 0.12 \\
\hline 25998 & 0.46 & F7 V & 29.54 & 2 & 33.93 & 156.25 \\
\hline 26923 & 0.59 & G0 V & 29.21 & 7 & 33.69 & 12.76 \\
\hline 29645 & 0.57 & G3 V & & 17 & 33.72 & 2.16 \\
\hline 30495 & 0.63 & G1 V & 28.83 & 11 & 33.61 & 5.17 \\
\hline 33608 & 0.46 & F6 V & 29.15 & 3 & 33.93 & 69.44 \\
\hline 35296 & 0.53 & $\mathrm{~F} 8 \mathrm{~V}$ & 29.44 & 4 & 33.78 & 39.06 \\
\hline 39587 & 0.59 & G0 V & 29.08 & 5 & 33.69 & 25.0 \\
\hline 43587 & 0.61 & G0 V & & 20 & 33.64 & 1.56 \\
\hline 45067 & 0.56 & F8 V & & 8 & 33.73 & 9.77 \\
\hline 61421 & 0.42 & F5 IV-V & 28.28 & 3 & 34.08 & 69.44 \\
\hline 72905 & 0.62 & F7 V & 29.11 & 5 & 33.63 & 25.00 \\
\hline 75332 & 0.49 & F7 V & 29.56 & 4 & 33.87 & 39.06 \\
\hline 76151 & 0.67 & G3 V & 28.33 & 15 & 33.52 & 2.78 \\
\hline 88335 & 0.46 & $\mathrm{~F} 6 \mathrm{~V}$ & & 5 & 33.93 & 25.00 \\
\hline 88737 & 0.56 & F5 V & & 8 & 33.73 & 9.77 \\
\hline 89744 & 0.54 & F6 V & & 9 & 33.77 & 7.72 \\
\hline 95735 & 1.51 & M2.1 Ve & 26.78 & 53 & 32.50 & 0.22 \\
\hline 97344 & 0.61 & G0 V & & 8 & 33.64 & 9.77 \\
\hline 100563 & 0.46 & F5 V & 29.13 & 4 & 33.47 & 39.06 \\
\hline 101501 & 0.72 & G8 V & 28.21 & 17 & 33.47 & 2.16 \\
\hline 106516 & 0.46 & F6 V & 27.93 & 7 & 33.93 & 12.76 \\
\hline 107213 & 0.50 & F8 V & & 9 & 33.85 & 7.72 \\
\hline
\end{tabular}


Table 4: Table 3. (Contd.)Stars with irregular (chaotic) chromospheric actifity

\begin{tabular}{ccclclc}
\hline HD & B-V & Spectrum & $\log L_{X}, \mathrm{erg} / \mathrm{s}$ & $P_{\text {rot },}, \mathrm{l}$ & $\log L_{\text {bol }}, \mathrm{erg} / \mathrm{s}$ & $D / D_{\odot}$ \\
\hline 114378 & 0.45 & F5 V+F5 V & 29.34 & 3 & 33.95 & 69.44 \\
115043 & 0.60 & G1 V & 28.05 & 6 & 33.66 & 17.36 \\
115383 & 0.58 & F8 V & 29.51 & 3 & 33.70 & 69.44 \\
115617 & 0.71 & G6 V & & 29 & 33.48 & 0.74 \\
124570 & 0.54 & F6 V & & 26 & 33.77 & 0.92 \\
124850 & 0.52 & F7 IV & 29.63 & 7 & 33.79 & 12.76 \\
126053 & 0.63 & G3 V & & 22 & 33.61 & 1.29 \\
129333 & 0.61 & G0 V & 30.01 & 3 & 33.64 & 69.44 \\
131156 A & 0.76 & G8 V & 28.90 & 6 & 33.42 & 17.36 \\
$131156 B$ & 1.17 & K4 V & 28.34 & 11 & 33.10 & 5.17 \\
136202 & 0.54 & F8 IV-V & & 14 & 33.77 & 3.19 \\
137107 AB & 0.58 & G2 V+G2 V & 28.41 & 14 & 33.70 & 3.19 \\
141004 & 0.60 & G0 V & 27.66 & 26 & 33.66 & 0.92 \\
142373 & 0.56 & F9 V & & 15 & 33.73 & 2.78 \\
143761 & 0.60 & G2 V & & 17 & 33.66 & 2.16 \\
155885 & 0.86 & K0 V & 28.28 & 21 & 33.35 & 1.42 \\
158614 & 0.72 & G8 IV-V V & & 34 & 33.47 & 0.54 \\
159332 & 0.48 & F4 V & & 7 & 33.89 & 12.76 \\
165341 & 1.16 & K6 V & 28.38 & 34 & 33.13 & 0.54 \\
176095 & 0.46 & F5 V & 29.00 & 4 & 33.93 & 39.06 \\
178428 & 0.70 & G4 V & 28.23 & 22 & 33.49 & 1.29 \\
182572 & 0.77 & G8 IV & 27.59 & 41 & 33.42 & 0.37 \\
185144 & 0.80 & K0 V & 27.61 & 27 & 33.40 & 0.86 \\
187013 & 0.47 & F5 V & 28.68 & 6 & 33.90 & 17.36 \\
190360 & 0.73 & G8 V & & 38 & 33.46 & 0.43 \\
207978 & 0.42 & G6 IV & & 3 & 34.08 & 69.44 \\
212754 & 0.52 & F0 V & & 12 & 33.79 & 4.34 \\
216385 & 0.48 & F7 IV & & 7 & 33.89 & 12.76 \\
217014 & 0.67 & G5 V & & 37 & 33.52 & 0.46 \\
\hline
\end{tabular}


of real discreteness associated with an increase in the number of half waves. An increase in the number of degrees of freedom of a dynamical system usually leads to a qualitative change in its behavior. In the case of a small number of degrees of freedom (modest dynamo number), the nonlinear evolution of the dynamical system has the character of self-excited oscillations (the system has extreme cyclicity). As the number of degrees of freedom grows, a regime of chaotic behavior in the form of strange attractors usually arises. We suggest that the numerous cases of aperiodic variations of stellar activity are associated with large dynamo numbers, with their corresponding chaotic regimes. The transition from extreme cyclicity to strange attractors and chaotic behavior represents a modern scenario for the development of turbulent flow from laminar flow; at its base lies a bifurcation that increases the dimension of the corresponding dynamical system. We propose that the appearance of discrete classes of stellar activity has analogous origins. In other words, we propose to interpret the fact that stars with well-defined cycles display comparatively low-intensity magnetic-field generation, while stars with more intensive field generation do not have well defined cycles, as observational confirmation of a "cyclic behavior-chaotic behavior" bifurcation in the stellar dynamo, associated with an increase in the effective dimension of the system as the dynamo number is increased. A possible alternative interpretation is that, in the case of large dynamo numbers, the magnetic field in the depths of the convective zone preserves cyclic behavior, but, for some reason, the link between the magnetic field and surface activity tracers is disrupted. We believe that this point of view encounters more difficulties, since the overall level of activity is increased when the dynamo number is increased, and only its cyclic character disappears. In connection with our proposed interpretation, it is valuable to consider the position of the Sun in these diagrams separately. Of course, the assignment of the Sun to the ranks of stars with very well defined activity periods is due to the anomalously small distance of the Sun from the Earth, and the associated exceptionally detailed study of solar activity that has been possible. It is well known that the intensities of different solar activity cycles can differ substantially; observing two such cycles on a distant star, it is doubtful that we would classify its cyclic activity as ideal. On the other hand, to a first approximation, the Hale polarity law is fulfilled on the Sun; i.e., data on sunspots specify in each hemisphere only a single half-wave of solar activity. At the same time, data for other tracers of solar activity specify additional waves, such as the polar wave, visible in analyses of polar facculae. Finally, 
the time dependence of solar activity reveals a well defined periodic component with an added chaotic component in the form of rare long-term drops in activity such as the Maunder minimum. All these data are consistent with the idea that the position of the Sun in our diagrams is near the edge of the region of well-defined periodic behavior.

\section{Conclusions}

Thus, among late-type stars, the soft X-ray luminosities and ratios $L_{X} / L_{b o l}$ are substantially higher for stars with irregular activity than for those with well defined cycles. This is consistent with the result obtained earlier in [12]. A detailed analysis for stars with reliably distinguished activity cycles indicates that, for comparatively slowly rotating stars of spectral types later than G0, the power of the corona decreases with the development of well defined cyclicity. This conclusion does not pertain to flare stars, for which a new effective mechanism for coronal heating probably begins to operate, associated with the occurrence of numerous weak flares (microflaring). The influence of age on the X-ray flux can be traced in our sample, however it is not strong for these solarneighborhood stars, and cannot be responsible for the observed variations of $L_{X}$ and $L_{X} / L_{b o l}$. Our conclusions are relevant for stars with spectral types from F5 to M3, in which the thickness of the surface convective zones is neither very small, nor very large compared to the stellar radius. This is consistent with the idea that realization of the dynamo mechanism requires the existence of an envelope in which the magnetic-field amplification can develop. It goes without saying that efficient operation of the dynamo mechanism requires differential rotation and turbulent convection. Our analysis demonstrates that the level of X-ray emission is primarily determined by the parameter that also determines the efficiency of the dynamo mechanism-the dynamo number. In the stars considered here, the influence of factors connected with the structure of their convective zones compensate each other to a large extent, so that, in accordance with (3), the dynamo number is determined by the square of the speed of axial rotation. The transition from small to large dynamo numbers gives rise to a change in the character of the dynamo process, increasing its chaotic component. We note especially that the general dependence of the activity on Rossby number discovered earlier is also preserved, due to the strong influence of 
the speed of axial rotation on both characteristic numbers-the Rossby and dynamo numbers. The behavior we have found has a more direct relationship to the theory of the $(\alpha-\Omega)$ dynamo. This behavior can be disrupted in stars with anomalously weak differential rotation, such as members of close binary systems, whose differential rotation is damped by tidal forces. In conclusion, we note that these results prompt us to re-evaluate the position of solar activity among analogous processes occurring on other stars. If we do not consider the exceptional star V2292 Oph, the Sun is located at the boundary of the region in Fig. 2a occupied by stars with cyclic activity, with the Sun rotating more rapidly than the other stars with well defined cycles. Note that the Sun's level of chromospheric activity is fairly high: the ratio of the luminosity in chromospheric emission to the bolometric luminosity is roughly the same as that of other stars with well defined cycles (see, for example, [4]). On the other hand, the solar corona is appreciably weaker than the typical coronas of active late-type stars: the solar ratio $L_{X} / L_{b o l}$ is about $10^{-7}$, while this ratio for other active stars on the lower part of the main sequence is several orders of magnitude higher.

\section{References}

[1] Wilson O. C. // Astrophys. J., 138, 932 (1968).

[2] Wilson O. C. // Astrophys. J., 226, 379 (1978).

[3] Baliunas S. L., Donahue R. A., Soon W. H. et al. // Astrophys. J., 438, 269 (1995).

[4] Radick R. R., Lockwood G. W., Skiff B. A., and Baliunas S. L. // Astrophys. J. Suppl. Ser., 118, 239 (1998).

[5] Alekseev I. Yu., and Gershberg R. E. // Astron. Rep., 40, 538 (1996).

[6] Alekseev I. Yu. // Astron. Rep., 44, 696 (2000).

[7] Hunsch M., Schmitt J. H. M. M., and Voges W. // Astron. Astrophys., Suppl. Ser., 132, 155 (1998).

[8] Hunsch M., Schmitt J. H. M. M., Sterzik M. F. ,and Voges W. // Astron. Astrophys., Suppl. Ser., 135, 319 (1999). 
[9] Vilhu O., and Walter F. M. // Astrophys. J., 321, 958 (1987).

[10] Gudel M., Guinan E. F. and Skinne S. L. // Astrophys. J., 483, 947 (1997).

[11] Katsova M. M., Badalyan O. G. and Livshits M. A. // Sov. Astron., 64, 1243 (1987).

[12] Hempelmann A., Schmitt J. H. M. M., and Stepien K. // Astron. Astrophys. , 305, 284 (1996).

[13] Straizis V., and Kuriliene G. // Astrophys. Space Sci. , 80, 353 (1981).

[14] Gershberg R. E., Katsova M. M., Lovkaya M. N. et al. // Astron. Astrophys., Suppl. Ser., 139, 555 (1999).

[15] Schussler M., Caligari P., Ferriz-Mas A. et al. // Astron. Astrophys., 314, 503 (1996).

[16] Vaughan A. H., and Preston R. W.// Publ. Astron. Soc. Pac., 92, 385 (1980).

[17] Noyes R. W., Hartmann L, W, Baliunas S. L. et al. // Astrophys. J, 279, 763 (1984).

[18] Baliunas S. L., Sokoloff D. D., and Soon W. // Astrophys. J, 279, 763 (1984).

[19] Acton L. W.// in The Cool Stars, Stellar Systems, and the Sun, Ed. by R. Pallavicini and A. K. Dupree, Astron. Soc. Pac. Conf. Ser. 109, 45 (1996).

[20] Pallavicini R. // Astron. Astrophys. Rev., 1, 177 (1989).

[21] Alekseev I. Yu., and Gershberg R. E., Katsova M. M., and Livshits M. A. // Astron. Rep., 45, 482 (2001).

[22] Gudel M., Linsky J. L., Brown A., and Nagase F., // Astrophys. J. 511, 405 (1999).

[23] Baliunas S. L., Nesme-Ribesand E., Nagase F., Sokoloff D., and Soon W. // Astrophys. J. 460, 848 (1996).

[24] Belvedere G., Lanza A., and Sokoloff // Sol. Phys. 183, 435 (1998). 